\title{
“Focused Ultrasound-mediated Drug Delivery in Humans - a Path Towards Translation in Neurodegenerative Diseases"
}

\author{
Joanna M. Wasielewska ${ }^{1,2}$ (D) Anthony R. White ${ }^{1,3}$ (D) \\ Received: 12 December 2021 / Accepted: 31 January 2022 / Published online: 7 March 2022 \\ (c) The Author(s) 2022
}

\begin{abstract}
The blood-brain barrier (BBB) has a major protective function in preventing the entry of harmful molecules into the brain, but is simultaneously limiting the delivery of drugs, restricting their potential clinical application in neurodegenerative diseases. Recent preclinical evidence demonstrates that following application of focused ultrasound with microbubbles (FUS $+\mathrm{MB}$ ), the BBB becomes reversibly accessible to compounds that normally are brain-impermeable, suggesting FUS+MB as a promising new platform for delivery of therapeutic agents into the central nervous system. As a step towards translation, small cohort clinical studies were performed demonstrating safe BBB opening in Alzheimer's disease, Parkinson's disease and amyotrophic lateral sclerosis (ALS) patients following FUS+MB, however improved drug delivery has not yet been achieved in human. Simultaneously, rapid progress in the human induced pluripotent stem cell (hiPSC) modeling technology allowed for development of novel Alzheimer's disease patient-derived BBB in vitro model that reacts to FUS+MB with BBB opening and can be used to answer fundamental questions of human BBB responses to FUS+MB in health and disease. This review summarizes key features of the BBB that contribute to limited drug delivery, recapitulates recent advances in the FUS + MB mediated human BBB opening in vivo and in vitro in the context of neurodegenerative disorders, and highlights potential strategies for fast-track translation of the FUS+MB to improve bioavailability of drugs to the human brain. With safe and effective application, this innovative FUS+MB technology may open new avenues for therapeutic interventions in neurodegenerative diseases leading to improved clinical outcomes for patients.
\end{abstract}

KEYWORDS Blood-brain barrier · Neurodegenerative disease $\cdot$ Focused ultrasound · Drug delivery $\cdot$ Human-induced pluripotent stem cell

Key Points

- The blood-brain barrier (BBB) is a multicellular structural barrier formed in brain capillaries that restricts the entry of most therapeutic agents into the central nervous system, posing the main challenge in treating neurodegenerative disorders.

- Application of focused ultrasound together with microbubbles has shown excellent potential in drug delivery across the BBB in preclinical models of neurodegeneration.

- Initial small cohort clinical trials showed safety and efficacy of ultrasound-mediated BBB opening in Alzheimer's disease, Parkinson's disease and amyotrophic lateral sclerosis (ALS), however improved drug delivery has not yet been achieved in neurodegenerative disease patients.

- Human in vitro BBB models can serve as an important platform accelerating clinical translation of focused ultrasoundmediated drug delivery in neurodegenerative disorders.

Joanna M. Wasielewska

Joanna.Wasielewska@qimrberghofer.edu.au

Extended author information available on the last page of the article

\section{Introduction}

\section{The Blood-brain Barrier as a Major Obstacle to Drug Delivery in Neurodegenerative Diseases}

The blood-brain barrier (BBB) formed in all cerebral capillaries is a dynamic multicellular interface that controls the exchange of molecules between the blood and the brain parenchyma [1]. By separating the circulating blood from the brain, it protects the central nervous system (CNS) from harmful molecules and pathogens, while at the same time maintaining tightly regulated brain homeostasis. Although essential for brain functioning, the BBB limits the ability of therapeutic agents to penetrate into the CNS, representing a major challenge in the treatment of neurodegenerative disorders [2].

The BBB is primarily formed by the specialised brain microvascular endothelial cells (BMECs) closely connected 
through tight junctions (TJs), adherent junctions (AJs), and gap junctions (GJs) [1]. At the molecular level, those junctional complexes are organised into sophisticated structures involving transmembrane proteins (such as claudins, occludins and junctional adhesion molecules (JAMs)) and numerous auxiliary proteins (as zonula occludens proteins, ZO-1, ZO-2 and ZO-3) that link adjacent BMECs and limit the para-cellular permeability of the BBB [1]. In contrast to other vascular endothelial cells found in the peripheral organs, specialised BMECs lack fenestrations (with only $4 \mathrm{~nm}$ wide extracellular gaps limited by TJs being present between BMECs as compared to $50 \mathrm{~nm}$ wide intracellular gaps in peripheral endothelium). The BBB endothelial cells also significantly restrict transcytosis (with BMECs containing scarce $1-15$ vesicles $/ \mu \mathrm{m}^{2}$ compared to $30-40$ vesicles/ $\mu \mathrm{m}^{2}$ in the peripheral EC), further contributing to minimal permeability of the BBB [3-6].

Other specialised components of the BBB include pericytes, astrocytes, adjacent neurons and non-cellular basement membrane, which together with BMECs form a socalled neurovascular unit (NVU) [1]. All elements of the NVU play an essential role in maintaining the BBB integrity and function. Pericytes are a member of the vascular smooth muscle cell lineage that regulate flow of blood in brain capillaries and contribute to the maintenance of TJs and AJs between adjacent BMECs. Astrocyte end-feet cover up to $99 \%$ of the BMEC surface and secrete glial-derived factors (such as glial cell line-derived neurotrophic factor (GDNF)) and morphogens (as retinoic acid or sonic hedgehog (Shh)) which support BMECs and improve the tightness of the BBB [7, 8]. The BBB is also in close connection with dendrites originating from local neurons that participate in the neurovascular coupling - a process essential for adequate blood supply to brain regions of increased neural activity. Finally, cells forming the NVU are embedded in the basement membrane - a unique form of thick extracellular matrix formed by fibronectin, collagen type IV, laminin and proteoglycan that supports the integrity and signalling at the BBB. These described properties and multicellular organisation result in a very high trans-endothelial resistance of the BBB reaching up to $5,900 \mathrm{Ohm} / \mathrm{cm}^{2}$ in vivo (as compared to $20 \mathrm{Ohm} / \mathrm{cm}^{2}$ measured in peripheral capillaries), forming one of the tightest barriers in the body [9-11].

Therefore, physiologically, only $\mathrm{O}_{2}, \mathrm{CO}_{2}$, water and small lipophilic molecules can pass through the BBB via simple diffusion, whereas the transport of most molecules is regulated by the expression of selective transporters at the surface of the BMECs [2]. Examples include solute carrier (SLC) transporters such as GLUT-1 (a glucose transporter), LAT-1 (an amino acid transporter), DMT-1 (divalent metal transporter 1) or SMVT (sodium dependent multivitamin transporter) that allow for controlled transport of essential nutrients, vitamins and ions between the blood and the CNS
[12]. Importantly, BMECs are also equipped with specialised efflux transporters that actively remove harmful metabolites and xenobiotics from the BMEC cytoplasm back to the blood, but simultaneously prevent the majority of drugs from entering the CNS. Correspondingly, expression of over 15 drug efflux transporters have been identified in BMECs, including multidrug resistance transporter (MDR1) and P-glycoprotein protein (P-gp), which together with the TJs, limit effective transport of therapeutics targeting the brain [13-16]. Finally, BMECs contain a variety of drug-metabolizing enzymes, that degrade or chemically modify drugs leading to their inactivation [17]. The aforementioned structural and molecular characteristics of the BBB significantly restrict successful drug delivery in the context of brain disease, limiting therapeutic potential of promising preclinical drug candidates. Importantly, vast evidence indicates that different mechanisms of molecular transport at the BBB may be altered during neurodegeneration, which further affects the distribution of therapeutics in the brain and decreases treatment efficiency [18]. Therefore, the development of innovative drug delivery methods is an urgent medical need to facilitate successful outcomes in treating neurodegenerative disorders.

\section{Focused Ultrasound as an Innovative Approach to Opening the Blood-brain Barrier}

With the BBB being the major challenge in the delivery of therapeutics for treating CNS disorders, several different strategies have been proposed to overcome this structural and functional barrier. One of them is an application of focused ultrasound (FUS) which, in combination with gasfilled microbubbles (MB), leads to reversible BBB opening and consequent improvements to drug bioavailability in the brain (Fig. 1). Since the initial discovery of its biological effect [19], vast progress has been made in the preclinical validation and technical development of FUS, leading to the initiation of clinical trials investigating application of therapeutic ultrasound in neurodegenerative disorders. Given the important advantages of FUS over other drug delivery methods such as non-invasiveness, spatial and temporal precision, reversibility and promising additional therapeutic effects [20-23], FUS holds the potential to emerge as an innovative multimodal tool for the treatment of neurodegenerative disorders.

\section{Current Understanding of Focused Ultrasound and Microbubble Effects on Brain Vasculature}

Successful clinical application of FUS + MB requires an in depth understanding of how its physical parameters translate 


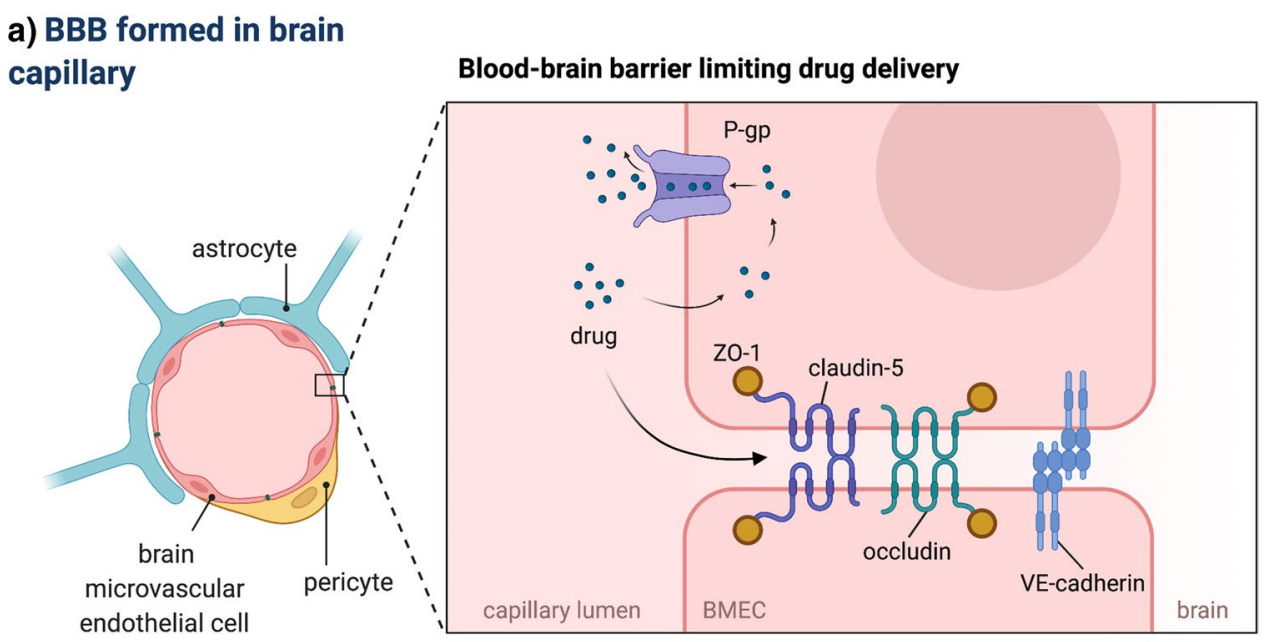

\section{b) Brain capillaries exposed to FUS+MB}

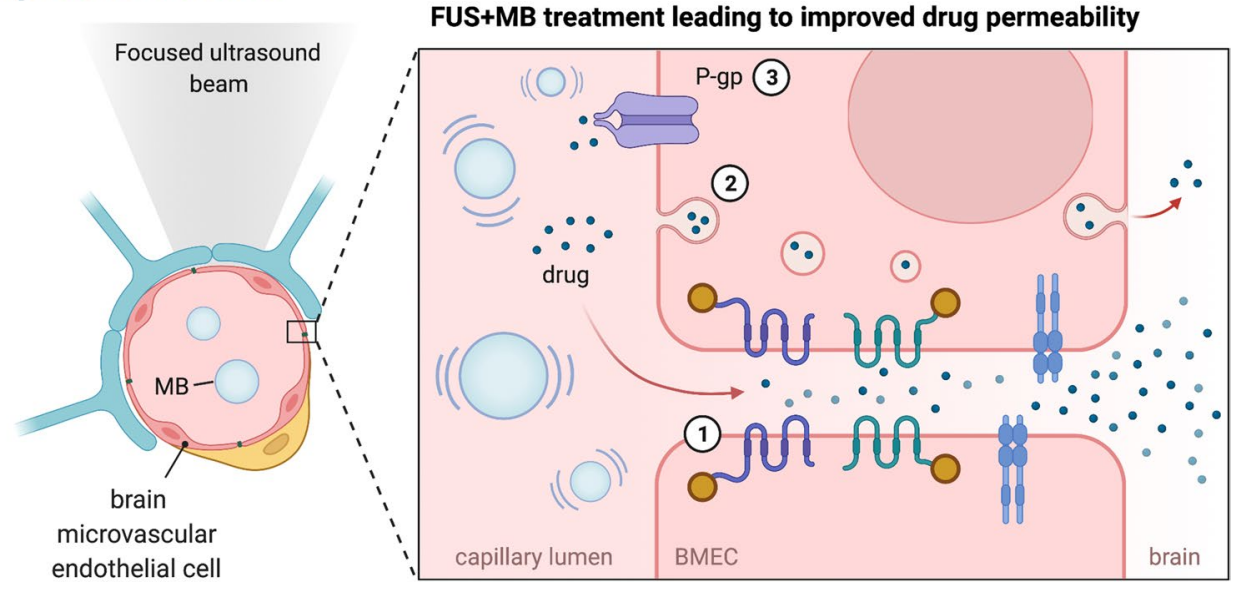

Fig. 1 Proposed mechanisms of focused ultrasound and microbubble mediated drug delivery at the blood-brain barrier. (a) Physiologically, the blood-brain barrier (BBB) formed by brain microvascular endothelial cells (BMEC), pericytes and astrocytes restricts the permeability of delivered drugs from the blood into the brain. The presence of tight- and adherens junctions containing transmembrane adhesion proteins such as vascular endothelial (VE)-cadherin, occludin, claudin-5 and zonula occludens-1 (ZO-1) accessory protein prevents paracellular transport of most hydrophilic molecules. Efflux transporters as P-glycoprotein (P-gp) actively remove a wide range of drugs from the BMEC cytoplasm, including small $(<400 \mathrm{Da})$ lipophilic molecules that might otherwise passively diffuse across BMEC to brain parenchyma. Transcytosis of molecules through the BBB is largely limited, significantly impeding the entry of therapeutic agents into the brain. (b) Preclinical observations identify three proposed routes of focused ultrasound and microbubble (FUS+MB) mediated drug delivery at the BBB. (1) MB oscillating in the ultrasonic field produce mechanical forces on the of tight- and adherens junctions, leading to temporal junction opening and improved paracellular transport of delivered drug. (2) FUS+MB treatment causes increase in the number of intracellular vesicles and upregulation of endo- and transcytosis at BMEC suggesting stimulation of transcellular transport at the BBB. (3) Exposure to FUS+MB temporarily (48-72 h) suppresses expression of P-gp at BMEC, potentially limiting drug efflux at the BBB. [1, 28, 31, 32, 118, 119]. BBB-blood-brain barrier; BMEC- brain microvascular endothelial cell; FUS+MB- focused ultrasound and microbubble; P-gp- P-glycoprotein; ZO-1-zonnula occludens; VE-cadherinvascular endothelial cadherin; Figure created with BioRender.com.

to the desired biological effect in the human brain. Current evidence coming from preclinical studies suggests that the BBB opening effect is achieved by the complex interactions of microbubbles and the vasculature in the ultrasonic field, with the extent of opening dependent on applied ultrasound parameters (frequency, acoustic pressure, burst repetition frequency, burst length, sonication duration) and microbubble properties (chemical formulation, concentration, size distribution, half-life) [24-27] (Fig. 2). When exposed to the ultrasound at low pressure, MB are known to oscillate volumetrically (expand and contract) in response to cycles of compression and rarefaction. This MB behavior termed stable cavitation produces mechanical effects on BMECs lining brain capillaries, including microstreaming (which is streaming flow of fluid/blood around an oscillating MB) and increased shear stress, that in turn creates tension at TJs and leads to increased vascular permeability [24]. Oscillating MB have also been shown to activate BMEC cell surface 

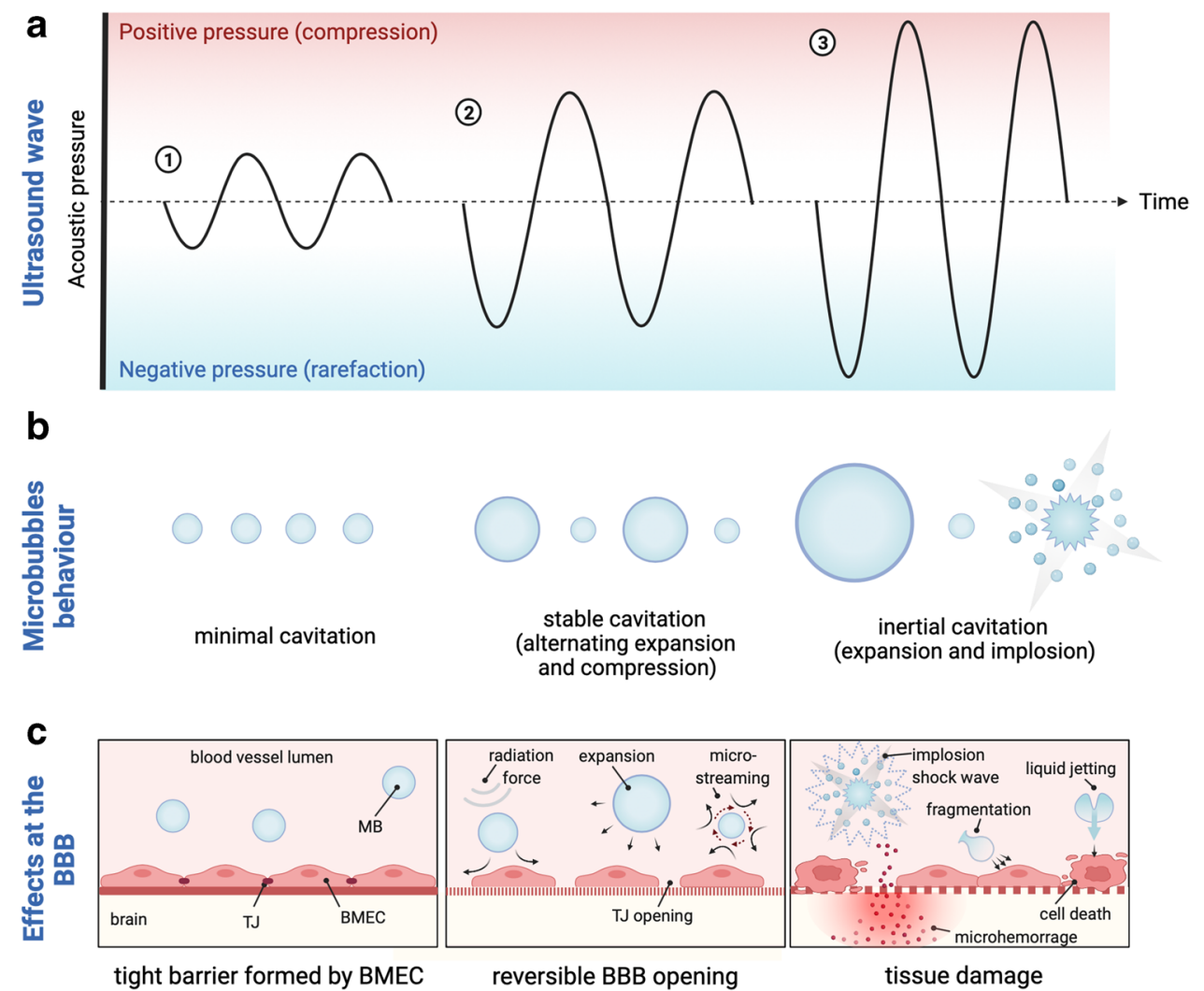

Fig. 2 The interplay between focused ultrasound, microbubbles and cerebral vasculature. Physical interactions between ultrasonic wave (a) and microbubbles (MB) (b) determine the bioeffects at the blood-brain barrier (BBB) (c). When exposed to the ultrasound wave, MB decrease in diameter during the compression portion of the wave and increase during the rarefaction phase. (1) Ultrasound applied at insufficient acoustic pressure causes minimal volumetric oscillations of MB and the BBB remains closed. (2) Optimal ultrasound acoustic pressure induces stable MB contraction and expansion (stable cavitation) that exerts mechanical forces on brain microvascular endothelial cells (BMEC), leading to reversible $\mathrm{BBB}$ opening. Linearly cavitating $\mathrm{MB}$ generate the flow of liquid/blood around themselves (microstreaming) that in turn produces sheer stress on BMEC membrane, causing increased BBB permeability. Expanding MB create tension at tight junction (TJ) proteins leading to junction opening. Acoustic radiation force propels oscillating MB to the BMEC layer, further enhancing MB and BBB interactions. (3) At higher acoustic pressures, $\mathrm{MB}$ collapse violently, producing shock waves and micro-jets. This abrupt inertial cavitation generates strong mechanical stress at the BBB leading to permanent TJ disruption, irreversible BMEC membrane perforation, microhemorrhage and tissue necrosis. [24, 25, 27, 33-35]. BBB-blood-brain barrier; BMEC- brain microvascular endothelial cell; MB-microbubble; TJ-tight junction; Figure created with BioRender.com.

receptors and downregulate the expression of efflux transporters (such as P-gp), and therefore modulate transcellular transport of large molecules at the BBB [28-32]. Ultrasound applied at excessive acoustic pressure however, causes rapid collapse of MB (termed as inertial cavitation) that can generate strong mechanical stresses, MB micro-jetting and thermal effects in the vasculature. This uncontrolled MB disruption underlies the majority of adverse effects observed during FUS-mediated BBB opening, including micro-hemorrhages, edema, glial scarring or tissue necrosis [33-35].

Given that MB behavior, and resulting bioeffects, are strongly dependent on the properties of the ultrasound wave, an in-depth understanding of sonication parameters is likely to drive safer application of FUS in humans. As such, although ultrasound frequencies ranging from $28 \mathrm{kHz}$ to $8 \mathrm{MHz}$ have been used to successfully to open BBB in animal models, for application in human brain the upper limit of $1.5 \mathrm{MHz}$ has been proposed to offer optimal focal volume of BBB opening with minimal (or no) focus aberration and tissue damage [36-38]. Secondly, given that the MB expansion and inertial cavitation are directly dependent on the peak positive and negative acoustic pressure (PNP) of the insonating wave, multiple studies investigated the relationship between PNP and vascular permeability [39, 40]. It has been shown that below a certain threshold, MB radial expansion and vascular leakage are positively correlated with PNP, however, further increase of the PNP leads to excessive inertial cavitation of the MBs and corresponding tissue damage [40, 41]. Although the interspecies differences (e.g. in skull acoustic attenuation) complicate direct translation of preclinical FUS parameters to human brain, it is now accepted that increasing acoustic pressure modulates the degree of BBB opening and/or adverse effects on the vasculature [42], and FUS at pressures at $0.2-1.0 \mathrm{MPa}(220$ 
$\mathrm{kHz}$ ) have been safely applied in small cohort clinical studies [43-46]. Finally, FUS burst-mode scheme including burst length, burst repetition frequency and sonication duration, although receiving less attention, have all been shown to affect biological responses to FUS+MB [47]. In this regard, a study presented by McDannold et al. reported positive correlation between FUS burst length and BBB permeability for bursts lasting $0.1-10 \mathrm{~ms}$ [24]. A further increase of burst length beyond $10 \mathrm{~ms}$ did not lead to increased BBB opening, possibly due to complete collapse of all MBs before the end of each burst $[24,48]$. Interestingly, increasing burst repetition frequency from $0.1 \mathrm{~Hz}$ to $1 \mathrm{~Hz}$, and therefore decreasing 10 -fold the sonication time needed to deliver the same number of bursts, also led to permeability enhancement [49, 50]. This effect has been attributed to the short half-life of MBs in the circulation (estimated $\sim 1.3$ min for commercially available $\mathrm{MB}$ ) and correspondingly reduced MB decay and larger number of MBs simultaneously oscillating during shorter FUS treatments. Importantly, Choi et al. observed also that the extent of BBB opening is affected by the length of the resting periods, where FUS bursts schemes including resting periods shorter than $0.1 \mathrm{~s}$ or longer than $1 \mathrm{~s}$ (for 1.5 $\mathrm{MHz}$ frequency) produced no effects on BBB permeability $[24,49]$.

Considerable progress has also been made in understanding how inherent MB properties affect the extent of BBB opening. Several studies reported a relationship between MB diameter and BBB permeability enhancement, where larger MBs were shown to induce a higher degree of BBB opening resulting in longer recovery time post treatment [51-53]. The extent of BBB opening was also positively correlated with the concentration of applied MBs [54]. Interestingly, Tung et al. showed that size-sorted MB with a diameter of 6-8 $\mu \mathrm{m}$ required lower acoustic pressure $(0.3$ $\mathrm{MPa}, 1.5 \mathrm{MHz})$, compared to MB with a diameter of 1-2 $\mu \mathrm{m}$, which required a higher acoustic pressure $(0.45 \mathrm{MPa}$, $1.5 \mathrm{MHz}$ ) to induce the same degree of BBB disruption [55]. Similar effects were observed by Choi et al. demonstrating close interplay between MB properties and applied FUS wave characteristics [56]. From a clinical perspective, all described parameters are of great relevance for successful and controlled drug delivery in the context of potential treatment for neurodegenerative disease.

\section{Focused Ultrasound Application in Neurodegenerative Disease Patients}

Despite extensive understanding of disease pathology and discovery of promising preclinical drug-candidates, brain disorders associated with profound neurodegeneration, including Alzheimer's disease (AD), Parkinson's disease dementia (PD) and amyotrophic lateral sclerosis (ALS), still have no cure. A primary obstacle linking all these disorders is the presence of the BBB limiting the transport of up $98-100 \%$ of administered drugs, preventing them from reaching therapeutic concentrations in the brain [57]. To overcome this hurdle, FUS+MB technology was recently evaluated in the first small cohort clinical studies opening a new avenue of investigation in the treatment of neurodegenerative diseases.

Lipsman et al. was the first to show BBB opening in a cohort of five $\mathrm{AD}$ patients using focused ultrasound and MB treatment [44]. In this seminal study, magnetic resonance-guided FUS (MRgFUS) was applied twice over the period of one month, targeting dorsolateral prefrontal cortex. Although no beneficial effects were found in amyloid burden or cognitive scores following sonication, the authors successfully demonstrated reversible and repeatable BBB opening in the absence of any adverse effects. Interestingly, transient changes in the resting state functional connectivity were observed post MRgFUS in patients, confirming possible neuromodulatory effects of FUS+MB reported in animal studies [21, 23, 58]. This pioneering work was followed by Rezai et al. who achieved FUS-mediated opening of the BBB in hippocampus and entorhinal cortex (EC) of six AD patients [59]. Enrolled patients tolerated a total of $17 \mathrm{MRgFUS}$ treatments with no adverse neurological or cognitive effects, demonstrating FUS+MS as a mean of non-invasive, reproducible, transient and spatially precise BBB opening. Interestingly, a follow up study revealed amyloid- $\beta$ plaque reduction following FUS+MB, suggesting promising therapeutic effects of FUS+MB in the absence of any additional drug treatment [60]. Subsequently, a clinical trial led by Mehta et al. confirmed feasibility of BBB opening in AD patients and identified a perivenular immunologic healing response downstream from the FUS+MB application, expanding our understanding of how FUS affects human vasculature [61].

Importantly, application of MRgFUS is expanding to other neurodegenerative diseases, with Abrahao and colleagues recently demonstrating the first evidence of successful BBB disruption in the motor cortex of four ALS patients [43] and Gasca-Salas et al. reporting safe BBB opening in $\mathrm{PD}$, targeting the right parieto-occipito-temporal cortex [45]. In both studies, no adverse side-effects were observed, with mild cognitive improvement being recorded post-treatment in PD patients. Interestingly, authors of all described clinical trials reported spontaneous closure of the BBB within $24 \mathrm{~h}$ post opening, suggesting $24 \mathrm{~h}$ as a clinically relevant drug administration timewindow, applicable to various types of neurodegenerative disorders. 


\section{Technical State-of-the-art of Blood-brain Barrier Opening in Human}

Promising clinical trial outcomes have motivated simultaneous technological progress leading to the development of clinical FUS brain devices and MB formulations. Currently available clinical prototype devices include extracorporeal low-frequency $(220-230 \mathrm{kHz}) \mathrm{MR}$-guided ExAblate Neuro Type 2 (InSightec, Israel) and neuronavigation-guided NaviFUS (Taiwan), as well as skullimplantable SonoCloud (CarThera, France), all allowing for non-thermal MB-mediated treatments [44, 62-64]. Although overcoming the problem of an ultrasound wave attenuation by the human skull, SonoCloud is currently the most invasive treatment. In this approach, the planar FUS transducer needs to be placed into a bur hole made on the patient's skull, targeting only one specific brain region that may not reflect more diffuse neurodegenerative disease pathological targets. Thus, the SonoCloud approach may be primarily limited to brain cancer treatment involving highly localized application [62, 65]. The ExAblate Neuro device, which has proven successful in all clinical trials to date [43-45, 59], integrates 1024 individual transducers with a frequency of $220 \mathrm{kHz}$ into a hemispherical helmet (Fig. 3a). Depending on the selected brain target location, the patient's skull properties, intraoperative MRI data, helmet orientation, and additional information (such as no-pass zones predicted for each patient), the system determines the necessary parameters for each individual transducer element to deliver precise FUS treatment to a prescribed target location. The device also integrates a dedicated single-element acoustic detector, allowing for real-time MB cavitation monitoring to support selection of sonication parameters for each individual procedure. The disadvantage of the system is that it requires intraoperative magnetic resonance imaging (MRI) to provide treatment guidance, therefore increasing associated cost, time and invasiveness for the patient.

Given physical and cognitive symptoms associated with neurodegenerative diseases and late disease onset for most of dementias, outpatient, non-invasive and relatively short FUS procedures must be considered to deliver treatment to the vast majority of patients. To achieve this goal, a frameless NaviFUS device (NaviFUS Inc.) incorporating a neuronavigation system is being currently tested, and has showed initial promising outcomes in recurrent glioblastoma patients [64]. Neuronavigation allows the guidance of the invisible FUS focal beam without intra-procedural imaging, as the patient's previous MRI or CT data are used to interactively visualize the position of applied FUS on a $3 \mathrm{D}$ anatomical brain image $[64,66]$. This promising technology has been approved for first clinical trials targeting Alzheimer's disease, possibly providing evidence of its utility in neurogenerative disorders. Additional
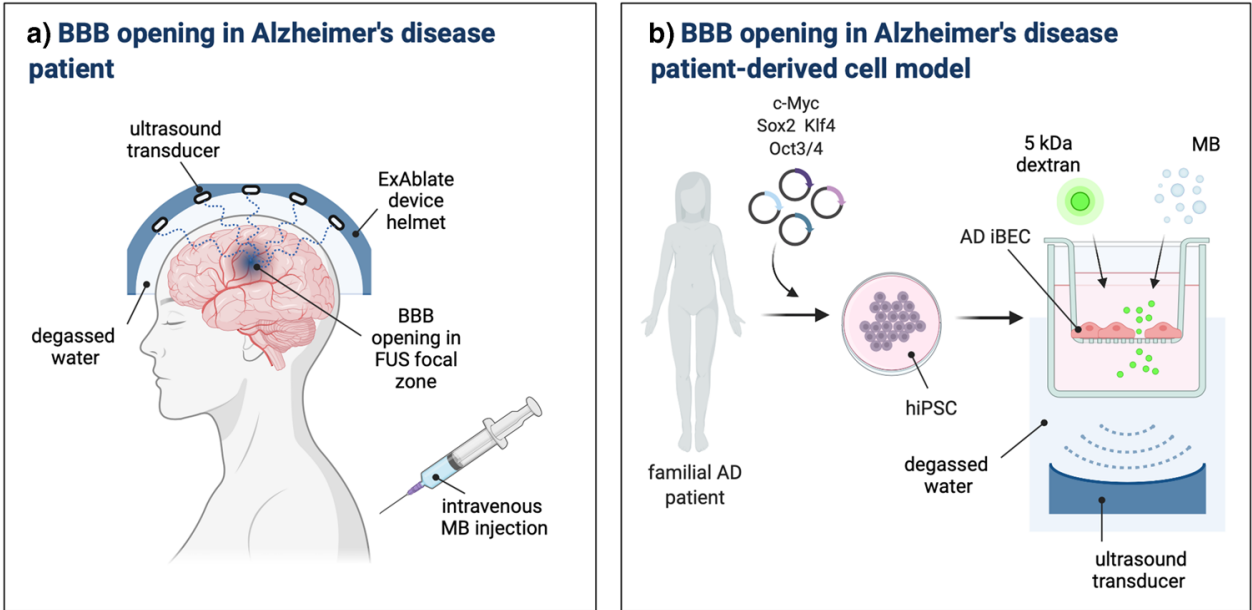

Fig. 3 Schematic representation of blood-brain barrier opening in Alzheimer's disease patient in vivo and patient-derived model in vitro. (a) Schematic of a magnetic resonance (MR)-guided ExAblate device used in the first successful blood-brain barrier (BBB) opening in Alzheimer's disease (AD) patients. System consists of a hemispherical helmet lined with $>1000$ independent transducer elements delivering low frequency ultrasound treatment to the prescribed target. The helmet is positioned in the specialised MRI bed with stereotaxic frame and the space between patient's head and the helmet filled with degassed water for acoustic coupling. Microbubble (MB) administration is carried out using repeated bolus injection or a continuous infusion. Reversible BBB opening occurs in the defined ultrasound focal zone. (b) Schematic of $\mathrm{AD}$ patient-derived human BBB opening in vitro. Somatic cells (e.g. fibroblasts or blood cells) are obtained from familial AD patients and reprogrammed to human induced pluripotent stem cells (hiPSC) by introduction of cocktail of reprogramming factors. hiPSC are used to generate brain endothelial-like cells (iBEC) and develop patient-derived in vitro AD BBB model. In vitro BBB is exposed to focused ultrasound and MB in the degassed water, leading to BBB opening and improved permeability of $5 \mathrm{kDa}$ dextran. [44, 84]. AD-Alzheimer's disease; BBB-bloodbrain barrier; FUS-focused ultrasound; hiPSC-human induced pluripotent stem cell; iBEC- brain endothelial-like cells; kDa-kilodalton; MBmicrobubble; Figure created with BioRender.com. 
FUS-devices originating from academic settings are also undergoing preclinical validation, expanding future treatment possibilities [67-69].

Microbubble formulations available in the clinic include FDA approved Definity (Lantheus Medical Imaging, USA), Optison (GEHealthcare,USA), and SonoVue (Bracco, Italy). The commercial MBs are sized 1-3 $\mu \mathrm{m}, 2-4 \mu \mathrm{m}$, and 2-5 $\mu \mathrm{m}$ respectively, offer a therapeutic application window of 5-10 min, and have different compositions and concentrations, allowing for control over desired biological effects. For example, larger MBs (i.e. SonoVue) were found to have a longer effective circulation time, facilitating extended sonication that may be required to deliver larger drugs such as therapeutic antibodies, however they were also associated with a higher risk of hemorrhage in preclinical studies [52, $54,55,70,71]$. Currently, the majority of clinical studies investigating effects of FUS in neurodegenerative diseases reported successful usage of lipid-shelled Definity (ExAblate) and SonoVue (NaviFUS) [44, 64], however continuous preclinical efforts in the MB development may lead to safer and more effective therapeutic applications of MB for BBB opening in patients.

With the safety window being sensitive to a multitude of technical factors, interdisciplinary research integrating biological and engineering perspectives may pave the way towards wide clinical application of FUS in neurodegenerative diseases.

\section{Focused Ultrasound Application in Human Blood-brain Barrier in vitro Models}

Despite the promising clinical success of $\mathrm{BBB}$ opening in $\mathrm{AD}, \mathrm{ALS}$ and PD, FUS+MB mediated drug delivery has not yet been trialed in patients due to many unanswered questions concerning interactions of human vasculature, ultrasound, and related drug transport dynamics.

An important step towards understanding the response of the human BBB to FUS+MB and accurate modeling of improved drug transport in various diseases may come from in vitro $\mathrm{BBB}$ models derived from human induced pluripotent stem cells (hiPSC). BBB models derived from hiPSCs have recently been shown to closely reflect in vivo drug permeability in the human brain [72,73]. With increasing availability of hiPSC lines generated from fibroblasts of patients suffering from neurodegenerative disorders and carrying disease-associated mutations (e.g. in genes including PSEN1 and APOE E4 in AD [74,75], C9orf72 and SOD1 in ALS [76], LRRK2 and SNCA in PD [77]) and constantly improving hiPSC differentiation protocols [78-83], in vitro BBB models may become a useful tool to study FUS-mediated drug transport. In fact, a recent study presented by Oikari et al. achieved human BBB opening in vitro with focused ultrasound, applying clinically relevant FUS parameters (Table 1) [84]. The authors developed an in vitro AD BBB model based on hiPSC-derived induced brain endothelial cells (iBEC) that responded to FUS+MB treatment with reversible $\mathrm{BBB}$ opening and increased permeability to a small molecule fluorescent tracer (Fig. 3b). Furthermore, iBECs carrying an AD-associated PSENI mutation showed longer recovery post treatment, identifying the first clinically relevant genotype-related difference in responses of human BBB to FUS+MB. Although drug delivery has not yet been trialed in this in vitro platform, promising results obtained with a $5 \mathrm{kDa}$ cargo molecule suggest suitability of this system for future semi-high throughput drug permeability screening.

Finally, extensive research indicates that pathological changes at the BBB in neurodegenerative diseases could have implications for drug transport [1]. Therefore, prior to broad clinical application, it is important to understand how FUS+MB could modulate the leakage kinetics of drugs in cerebral vasculature of patients suffering from distinct disorders. Patient-derived cells forming the NVU have been shown to maintain disease-associated phenotypes (BMECs [76, 84], pericytes [85], astrocytes [74, 86-88]). Therefore, investigation of FUS effects using in vitro models could help to optimise parameters accommodating pathological changes at the BBB characteristic to specific disease and/ or genotype, and understanding molecular mechanisms
Table 1 Clinically Relevant Ultrasound and MB Parameters for Blood-brain Barrier Opening in Neurodegenerative Disease Patients and in vitro Model of Neurodegeneration. [43-45, 84]

\begin{tabular}{lll}
\hline Parameter & In vivo human patients & In vitro human BBB model \\
\hline Center frequency & $220-230 \mathrm{kHz}$ & $286 \mathrm{kHz}$ \\
Peak negative pressure & $0.2-1.0 \mathrm{MPa}$ & $0.15-0.3 \mathrm{MPa}$ \\
Mechanical index & 0.4 & $0.3-0.6$ \\
Burst length & $10 \mathrm{~ms}$ & $20 \mathrm{~ms}$ \\
Sonication duration & $50 \mathrm{~s}-120 \mathrm{~s}$ & $120 \mathrm{~s}$ \\
Microbubble dose & $4-10 \mu \mathrm{L} / \mathrm{kg}$ (Definity) & $10 \mu \mathrm{L} /$ well (in-house MB) \\
Reference & {$[43-45]$} & {$[84]$} \\
\hline
\end{tabular}

BBB- blood-brain barrier; kHz- kilohertz; MB- microbubble; MPa-megapascal; ms- millisecond; s- second; 
responsible for FUS-mediated drug transport in brain dis orders. Application of FUS+MB to in vitro BBB models could also facilitate the analysis of sonication-associated effects at single cell resolution, otherwise not possible in the living human brain. Furthermore, with hundreds of potential new-drug candidates being investigated in pre-clinical and early clinical trials for the treatment of various neurodegenerative disorders, effective high-throughput in vitro screening methods are necessary to identify therapeutics most compatible with FUS-mediated delivery in humans. This in-depth understanding of patient-specific cellular and molecular responses to FUS may aid in the selection of the drug candidates and FUS parameters most suitable for specific patients and disease.

\section{Human in vitro BBB Models can Bridge the Gap Between FUS-mediated Drug Delivery in Preclinical Animal Models, and Successful Clinical Therapeutic Outcomes}

It is estimated that the brain capillary network in humans has a total length of $600 \mathrm{~km}$, providing $15-30 \mathrm{~m}^{2}$ surface of selectively permeable BBB and offering a highly attractive route of drug delivery into the brain in the context of disease [89].

FUS+MB has extensively shown to increase permeability of therapeutic agents in animal models of neurodegeneration including AD [90-93] and PD [94-97], leading to the reversal of some disease symptoms. With initial clinical trials showing safe BBB opening in humans [43-45], currently the biggest challenge is to use FUS+MB to achieve improved drug delivery in human patients in the absence of any adverse effects.

One of the first steps to facilitate this is to induce opening of the larger focal volume of the BBB in neurodegenerative disease patients. Currently, successful clinical trials report opening of $>1 \mathrm{~cm}^{3}$ of human BBB [43, 44], which given widespread pathology observed in neurodegenerative diseases, may not be sufficient to achieve meaningful therapeutic outcomes. However, since FUS+MB has been shown to induce proinflammatory responses in animal models [98] and age-associated blood-borne factors could be detrimental if entering the brain in excessive amounts [99, 100], opening of the BBB in the entire brain as achieved in FUS-scanning mode in mouse models $[20,92,101]$ can have adverse effects for the patient.

hiPSC-derived BBB models may become an important tool for simulation of potential adverse responses in human $\mathrm{BBB}$ to FUS+MB, and identify co-treatments or settings that could attenuate these effects. The high-throughput potential of novel human BBB models [83, 102] and applicability of FUS+MB in vitro [84] could also facilitate screening of various combinations of disease-targeting drug and adjuvant therapies alleviating effects of FUS in the context of distinct neurodegenerative diseases or underlying mutations, before clinical application. For example, glucocorticoids (GC) have known immunosuppressive effect and were shown to reduce brain edema and improve tightness of the BBB by upregulating expression of occludin, claudins and VE-cadherin [103]. Since iBECs carrying AD-associated PSENI mutation previously demonstrated prolonged recovery post FUS in vitro [84], GC could be trialed to reduce inflammation and support barrier closure in familial AD patients who could be at risk of extended BBB disruption. Such experiments could also predict potential adverse effects of e.g. local FUSinduced inflammation or molecular effects from particular drug uptake and metabolism allowing for effective selection of most promising drug candidates.

While moving to larger target opening volumes and drug delivery, the heterogeneity of the BBB in the brain must also be considered [104, 105].

Recent single-cell RNA-seq studies revealed molecular heterogeneity of endothelial and mural cells found within different vascular segments and brain regions [105-108]. This transcriptomic heterogeneity of the NVU may have an implication for regional responses to FUS+MB as for example, BMECs found in hippocampus showed enrichment in inflammatory response and cell death genes as compared to cortex BMECs, indicating higher susceptibility of the hippocampus to vascular inflammation and injury [105]. Therefore, it is possible that observed segmentation of the cerebrovascular tree can affect vascular responses to FUS and MB parameters with certain brain regions being more susceptible to FUS+MB induced damage.

The NVU has also been shown to vary in the cellular architecture across different regions with a recent report demonstrating specialised BBB composition in the hippocampus enriched in the neural stem cells (NSC) [109]. In this study, Licht and colleagues identified the presence of NSC apical processes within NVU of the murine dentate gyrus that created direct connections with BMEC and led to selective uptake of the otherwise BBB-impermeable chemotherapeutic agent doxorubicin from the blood stream. With observed cognitive impairment as a side-effect of doxorubicin treatment, it is possible the same mechanism occurs in human brain [110]. This suggests that the cellular heterogeneity of the BBB in the brain can translate into region specific drug uptake and may have important implications for FUS-mediated drug delivery. With the hippocampus being strongly implicated in the pathology of dementia and therefore offering an attractive drug target region, development of new brain area-specific BBB in vitro models, e.g. hippocampal BBB enriched in neural stem cell components, may help elucidate regional responses of the BBB to FUS+MB and 
facilitate the most effective drug delivery strategy. Another example comes from PD where neurodegeneration is driven by progressive dysfunction of dopaminergic neurons in the substantia nigra - an area projecting to the cerebral cortex which was targeted by FUS+MB opening in a PD clinical trial [111] and in rat brain [112]. Recently, Pediaditakis et al. developed a novel substantia-nigra human Brain-Chip in vitro model containing dopaminergic neurons, astrocytes, microglia, pericytes, and BMEC that could serve as a testing platform for validation of new drugs for PD [113]. Such multicellular brain-region specific BBB models, when combined with FUS+MB in vitro system, could become useful predictors of FUS-mediated therapeutic uptake in diseaseassociated brain structures.

Finally, neurodegenerative disorders are one of the most heterogenous disease types with broad interpatient variability observed in disease onset, clinical symptoms, pace of progression, genetic mutations underlying the disease, and resulting responsiveness to treatment $[114,115]$. This heterogeneity may be a key confound to understanding the patient's BBB responses to FUS+MB and impede identification of one-size-fits-all FUS+MB parameters leading to safe BBB opening. With increasing speed of hiPSC reprogramming $[116,117]$ and expanding availability of in vitro BBB models derived from AD, PD, ALS and Huntington's disease (HD) patient's hiPSCs [76, 84], the high-throughput screening of sonication and $\mathrm{MB}$ effects on vasculature in specific patient or disease models may aid in personalization of FUS+MB treatment limiting the risk of side effects. This biological experimentation when combined with rapid technological progress in ultrasound devices will allow for tailoring of FUS sonication, MB type and drug treatment individually for the needs of specific patients to maximize the therapeutic effects of ultrasound in the clinic.

\section{Conclusions}

FUS+MB mediated BBB opening, and consequent improved drug delivery, holds the promise to revolutionize neurodegenerative disease treatment.

Despite the profound preclinical and clinical progress in recent years, it still remains unknown whether localised BBB opening in human brain would indeed lead to increased drug uptake, how this would affect pharmacokinetics of drugs in the neurodegenerative brain environment, and if overall improved therapeutic outcomes will be achieved.

Novel patient-derived in vitro BBB models may aid in answering at least some of these questions, allowing for high-throughput FUS+MB parameters and drug treatment screening in the disease and genotype specific manner. Together, interdisciplinary efforts encompassing in vitro investigations, well designed clinical trials, and biomedical engineering will lead to safe and effective BBB opening in patients, paving the way towards successful translation of FUS+MB technology in neurodegenerative diseases.

Author contributions JMW: concept and original manuscript draft preparation, ARW: manuscript editing and review.

Funding Open Access funding enabled and organized by CAUL and its Member Institutions. A.R.W. is a recipient of an NHMRC Senior Research Fellowship (APP1118452).

Availability of data and material Not applicable.

Code availability Not applicable.

\section{Declarations}

Ethics approval Not applicable.

Consent to participate Not applicable.

Conflicts of interest Joanna M. Wasielewska and Anthony R. White declare that they have no conflict of interest.

Open Access This article is licensed under a Creative Commons Attribution 4.0 International License, which permits use, sharing, adaptation, distribution and reproduction in any medium or format, as long as you give appropriate credit to the original author(s) and the source, provide a link to the Creative Commons licence, and indicate if changes were made. The images or other third party material in this article are included in the article's Creative Commons licence, unless indicated otherwise in a credit line to the material. If material is not included in the article's Creative Commons licence and your intended use is not permitted by statutory regulation or exceeds the permitted use, you will need to obtain permission directly from the copyright holder. To view a copy of this licence, visit http://creativecommons.org/licenses/by/4.0/.

\section{References}

1. Sweeney MD, Zhao Z, Montagne A, Nelson A, Zlokovic B. Blood-brain barrier: from physiology to disease and back. Physiological Reviews. 2019;99(1):21-78.

2. Wasielewska JM, Da Silva Chaves JC, White AR, Oikari LE. Modeling the Blood-Brain Barrier to Understand Drug Delivery in Alzheimer's Disease. In: Huang X, editor. Alzheimer's Disease: Drug Discovery. Brisbane (AU): Exon Publications; 2020

3. Satchell SC, Braet F. Glomerular endothelial cell fenestrations: an integral component of the glomerular filtration barrier. Am J Physiol Renal Physiol. 2009 May;296(5):F947-56.

4. Bauer H-C, Krizbai IA, Bauer H, Traweger A. "You Shall Not Pass"-tight junctions of the blood brain barrier. Front Neurosci. 2014;8:392-392.

5. Stewart PA. Endothelial vesicles in the blood-brain barrier: are they related to permeability? Cell Mol Neurobiol. 2000 Apr;20(2):149-63.

6. Pulgar VM. Transcytosis to Cross the Blood Brain Barrier, New Advancements and Challenges. Frontiers in Neuroscience. 2019;12:1019. 
7. Abbott NJ, Rönnbäck L, Hansson E. Astrocyte-endothelial interactions at the blood-brain barrier. Nat Rev Neurosci. 2006 Jan;7(1):41-53.

8. Spampinato SF, Bortolotto V, Canonico PL, Sortino MA, Grilli M. Astrocyte-Derived Paracrine Signals: Relevance for Neurogenic Niche Regulation and Blood-Brain Barrier Integrity. Front Pharmacol. 2019;10:1346.

9. Olesen SP, Crone C. Electrical resistance of muscle capillary endothelium. Biophys J. 1983 Apr;42(1):31-41.

10. Butt AM, Jones HC, Abbott NJ. Electrical resistance across the blood-brain barrier in anaesthetized rats: a developmental study. J Physiol. 1990 Oct;429:47-62.

11. Crone C, Olesen SP. Electrical resistance of brain microvascular endothelium. Brain Res. 1982 Jun 3;241(1):49-55.

12. Campos-Bedolla P, Walter FR, Veszelka S, Deli MA. Role of the Blood-Brain Barrier in the Nutrition of the Central Nervous System. Arch Med Res. 2014 Nov 1;45(8):610-38.

13. Omidi Y, Campbell L, Barar J, Connell D, Akhtar S, Gumbleton M. Evaluation of the immortalised mouse brain capillary endothelial cell line, b.End3, as an in vitro blood-brain barrier model for drug uptake and transport studies. Brain Res. 2003 Nov 14;990(1-2):95-112.

14. Kusuhara H, Sugiyama Y. Active efflux across the bloodbrain barrier: role of the solute carrier family. NeuroRx. 2005 Jan;2(1):73-85.

15. Mahringer A, Fricker G. ABC transporters at the blood-brain barrier. Expert Opinion on Drug Metabolism \& Toxicology. 2016 May 3;12(5):499-508.

16. Poller B, Gutmann H, Krähenbühl S, Weksler B, Romero I, Couraud $\mathrm{P}-\mathrm{O}$, et al. The human brain endothelial cell line hCMEC/ D3 as a human blood-brain barrier model for drug transport studies. J Neurochem. 2008;107(5):1358-68.

17. Agúndez JAG, Jiménez-Jiménez FJ, Alonso-Navarro H, GarcíaMartín E. Drug and xenobiotic biotransformation in the bloodbrain barrier: a neglected issue. Front Cell Neurosci. 2014 Oct $17 ; 8: 335$.

18. Schenk GJ, de Vries HE. Altered blood-brain barrier transport in neuro-inflammatory disorders. Drug Discovery Today: Technologies. 2016 Jun 1;20:5-11.

19. Hynynen K, McDannold N, Vykhodtseva N, Jolesz FA. Noninvasive MR Imaging-guided Focal Opening of the Blood-Brain Barrier in Rabbits. Radiology. 2001 Sep 1;220(3):640-6.

20. Leinenga G, Goetz J. Scanning ultrasound removes amyloid-beta and restores memory in an Alzheimer's disease mouse model. Science Translational Medicine. 2015;7(278):278ra33.

21. Blackmore DG, Turpin F, Palliyaguru T, Evans HT, Chicoteau A, Lee W, et al. Low-intensity ultrasound restores long-term potentiation and memory in senescent mice through pleiotropic mechanisms including NMDAR signaling. Mol Psychiatry. 2021 May;27:1-17.

22. Shin J, Kong C, Lee J, Choi BY, Sim J, Koh CS, et al. Focused ultrasound-induced blood-brain barrier opening improves adult hippocampal neurogenesis and cognitive function in a cholinergic degeneration dementia rat model. Alzheimer's Research \& Therapy. 2019 Dec 27;11(1):110.

23. Mooney SJ, Shah K, Yeung S, Burgess A, Aubert I, Hynynen K. Focused Ultrasound-Induced Neurogenesis Requires an Increase in Blood-Brain Barrier Permeability. PLoS One. 2016 Jul 26;11(7):e0159892.

24. McDannold N, Vykhodtseva N, Hynynen K. Effects of acoustic parameters and ultrasound contrast agent dose on focused-ultrasound induced blood-brain barrier disruption. Ultrasound Med Biol. 2008 Jun;34(6):930-7.

25. Nhan T, Burgess A, Cho EE, Stefanovic B, Lilge L, Hynynen K. Drug delivery to the brain by focused ultrasound induced bloodbrain barrier disruption: quantitative evaluation of enhanced permeability of cerebral vasculature using two-photon microscopy. J Control Release. 2013 Nov 28;172(1):274-80.

26. O'Reilly MA, Jones RM, Barrett E, Schwab AP, Head E, Hynynen K. Investigation of the Safety of Focused UltrasoundInduced Blood-Brain Barrier Opening in a Natural Canine Model of Aging. 2017;7(14):3573-84.

27. Chen K, Wei K, Liu HL. Theranostic Strategy of Focused Ultrasound Induced Blood-Brain Barrier Opening for CNS Disease Treatment. Front Pharmacol. 2019;10:86.

28. Pandit R, Koh WK, Sullivan RKP, Palliyaguru T, Parton RG, Götz J. Role for caveolin-mediated transcytosis in facilitating transport of large cargoes into the brain via ultrasound. J Controlled Release. 2020 Nov 10;327:667-75.

29. Cho H, Lee H-Y, Han M, Choi J, Ahn S, Lee T, et al. Localized Down-regulation of P-glycoprotein by Focused Ultrasound and Microbubbles induced Blood-Brain Barrier Disruption in Rat Brain. Sci Rep. 2016 Aug 11;6:31201.

30. Choi H, Lee E, Han M, An S, Park J. Diminished Expression of P-glycoprotein Using Focused Ultrasound Is Associated With JNK-Dependent Signaling Pathway in Cerebral Blood Vessels. Front Neurosci. 2019;13:1350.

31. Aryal M, Fischer K, Gentile C, Gitto S, Zhang Y-Z, McDannold N. Effects on P-Glycoprotein Expression after Blood-Brain Barrier Disruption Using Focused Ultrasound and Microbubbles. PLoS One. 2017 Jan 3;12(1):e0166061.

32. Sheikov N, Mcdannold N, Sharma S, Hynynen K. Effect of Focused Ultrasound Applied With an Ultrasound Contrast Agent on the Tight Junctional Integrity of the Brain Microvascular Endothelium. Ultrasound in Medicine \& Biology. 2008;34(7):1093-104.

33. Fan C-H, Liu H-L, Huang C-Y, Ma Y-J, Yen T-C, Yeh C-K. Detection of Intracerebral Hemorrhage and Transient BloodSupply Shortage in Focused-Ultrasound-Induced Blood-Brain Barrier Disruption by Ultrasound Imaging. Ultrasound in Medicine and Biology. 2012 Aug 1;38(8):1372-82.

34. Liu H-L, Wai Y-Y, Chen W-S, Chen J-C, Hsu P-H, Wu X-Y, et al. Hemorrhage detection during focused-ultrasound induced blood-brain-barrier opening by using susceptibility-weighted magnetic resonance imaging. Ultrasound Med Biol. 2008 Apr;34(4):598-606.

35. Arvanitis CD, Vykhodtseva N, Jolesz F, Livingstone M, McDannold N. Cavitation-enhanced nonthermal ablation in deep brain targets: feasibility in a large animal model. J Neurosurg. 2016 May 1;124(5):1450-9.

36. Pajek D, Hynynen K. The application of sparse arrays in high frequency transcranial focused ultrasound therapy: A simulation study. Med Phys. 2013 Dec;40(12):122901.

37. Liu H-L, Pan C-H, Ting C-Y, Hsiao M-J. Opening of the BloodBrain Barrier by Low-Frequency (28-kHz) Ultrasound: A Novel Pinhole-Assisted Mechanical Scanning Device. Ultrasound in Medicine and Biology. 2010 Feb 1;36(2):325-35.

38. Bing KF, Howles GP, Qi Y, Palmeri ML, Nightingale KR. BloodBrain Barrier (BBB) Disruption Using a Diagnostic Ultrasound Scanner and Definity ${ }^{\circledR}$ in Mice. Ultrasound Med Biol. 2009 Aug;35(8):1298-308.

39. Hosseinkhah N, Goertz DE, Hynynen K. Microbubbles and Blood Brain Barrier Opening: A Numerical Study on Acoustic Emissions and Wall Stress Predictions. IEEE Trans Biomed Eng. 2015 May;62(5):1293-304.

40. Hynynen K, McDannold N, Martin H, Jolesz FA, Vykhodtseva $\mathrm{N}$. The threshold for brain damage in rabbits induced by bursts of ultrasound in the presence of an ultrasound contrast agent (Optison $\left.{ }^{\circledR}\right)$. Ultrasound in Medicine and Biology. 2003 Mar $1 ; 29(3): 473-81$.

41. McDannold N, Vykhodtseva N, Raymond S, Jolesz FA, Hynynen K. MRI-guided targeted blood-brain barrier disruption with 
focused ultrasound: Histological findings in rabbits. Ultrasound in Medicine and Biology. 2005 Nov 1;31(11):1527-37.

42. Song K-H, Harvey BK, Borden MA. State-of-the-art of microbubble-assisted blood-brain barrier disruption. Theranostics. 2018 Aug 7;8(16):4393-408.

43. Abrahao A, Meng Y, Llinas M, Huang Y, Hamani C, Mainprize $\mathrm{T}$, et al. First-in-human trial of blood-brain barrier opening in amyotrophic lateral sclerosis using MR-guided focused ultrasound. Nat Commun. 2019 Sep 26;10(1):4373.

44. Lipsman N, Meng Y, Bethune AJ, Huang Y, Lam B, Masellis $\mathrm{M}$, et al. Blood-brain barrier opening in Alzheimer's disease using MR-guided focused ultrasound. Nature Communications. 2018;9(1):1-8.

45. Gasca-Salas C, Fernández-Rodríguez B, Pineda-Pardo JA, Rodríguez-Rojas R, Obeso I, Hernández-Fernández F, et al. Bloodbrain barrier opening with focused ultrasound in Parkinson's disease dementia. Nat Commun. 2021 Feb 3;12(1):779.

46. Huang Y, Alkins R, Schwartz ML, Hynynen K. Opening the Blood-Brain Barrier with MR Imaging-guided Focused Ultrasound: Preclinical Testing on a Trans-Human Skull Porcine Model. Radiology. 2017 Jan;282(1):123-30.

47. Chopra R, Vykhodtseva N, Hynynen K. Influence of Exposure Time and Pressure Amplitude on Blood-Brain-Barrier Opening Using Transcranial Ultrasound Exposures. ACS Chem Neurosci. 2010 Mar 2;1(5):391-8.

48. O'Reilly MA, Waspe AC, Ganguly M, Hynynen K. FocusedUltrasound Disruption of the Blood-Brain Barrier Using CloselyTimed Short Pulses: Influence of Sonication Parameters and Injection Rate. Ultrasound Med Biol. 2011 Apr;37(4):587-94.

49. Choi JJ, Selert K, Gao Z, Samiotaki G, Baseri B, Konofagou EE. Noninvasive and localized blood-brain barrier disruption using focused ultrasound can be achieved at short pulse lengths and low pulse repetition frequencies. J Cereb Blood Flow Metab. 2011 Feb;31(2):725-37.

50. Goertz DE, Wright C, Hynynen K. Contrast Agent Kinetics in the Rabbit Brain During Exposure to Therapeutic Ultrasound. Ultrasound Med Biol. 2010 Jun;36(6):916-24.

51. Samiotaki G, Vlachos F, Tung Y-S, Konofagou EE. A quantitative pressure and microbubble-size dependence study of focused ultrasound-induced blood-brain barrier opening reversibility in vivo using MRI. Magn Reson Med. 2012 Mar;67(3):769-77.

52. Vlachos F, Tung Y-S, Konofagou E. Permeability dependence study of the focused ultrasound-induced blood-brain barrier opening at distinct pressures and microbubble diameters using DCE-MRI. Magnetic Resonance in Medicine. 2011;66(3):821-30.

53. McMahon D, Lassus A, Gaud E, Jeannot V, Hynynen K. Microbubble formulation influences inflammatory response to focused ultrasound exposure in the brain. Sci Rep. 2020 Dec 9;10(1):21534.

54. Yang F-Y, Fu W-M, Yang R-S, Liou H-C, Kang K-H, Lin W-L. Quantitative Evaluation of Focused Ultrasound with a Contrast Agent on Blood-Brain Barrier Disruption. Ultrasound in Medicine and Biology. 2007 Sep 1;33(9):1421-7.

55. Tung Y-S, Vlachos F, Feshitan JA, Borden MA, Konofagou EE. The mechanism of interaction between focused ultrasound and microbubbles in blood-brain barrier opening in mice. J Acoust Soc Am. 2011 Nov;130(5):3059-67.

56. Choi JJ, Feshitan JA, Baseri B, Wang S, Tung Y-S, Borden MA, et al. Microbubble-size dependence of focused ultrasoundinduced blood-brain barrier opening in mice in vivo. IEEE Trans Biomed Eng. 2010 Jan;57(1):145-54.

57. Pardridge WM. The Blood-Brain Barrier: Bottleneck in Brain Drug Development. NeuroRX. 2005;2(1):3-14.

58. Meng Y, MacIntosh BJ, Shirzadi Z, Kiss A, Bethune A, Heyn $\mathrm{C}$, et al. Resting state functional connectivity changes after
MR-guided focused ultrasound mediated blood-brain barrier opening in patients with Alzheimer's disease. NeuroImage. 2019 Oct 15;200:275-80.

59. Rezai AR, Ranjan M, D'Haese P-F, Haut MW, Carpenter J, Najib U, et al. Noninvasive hippocampal blood-brain barrier opening in Alzheimer's disease with focused ultrasound. PNAS. 2020 Apr 28;117(17):9180-2.

60. D'Haese P-F, Ranjan M, Song A, Haut MW, Carpenter J, Dieb $\mathrm{G}$, et al. $\beta$-Amyloid Plaque Reduction in the Hippocampus After Focused Ultrasound-Induced Blood-Brain Barrier Opening in Alzheimer's Disease. Frontiers in Human Neuroscience. 2020;14:422.

61. Mehta RI, Carpenter JS, Mehta RI, Haut MW, Ranjan M, Najib U, et al. Blood-Brain Barrier Opening with MRI-guided Focused Ultrasound Elicits Meningeal Venous Permeability in Humans with Early Alzheimer Disease. Radiology. 2021 Mar;298(3):654-62.

62. Carpentier A, Canney M, Vignot A, Reina V, Beccaria K, Horodyckid C, et al. Clinical trial of blood-brain barrier disruption by pulsed ultrasound. Sci Transl Med. 2016 Jun 15;8(343):343re2.

63. Idbaih A, Canney M, Belin L, Desseaux C, Vignot A, Bouchoux G, et al. Safety and Feasibility of Repeated and Transient BloodBrain Barrier Disruption by Pulsed Ultrasound in Patients with Recurrent Glioblastoma. Clinical cancer research : an official journal of the American Association for Cancer Research. 2019;25(13):3793-801.

64. Chen K-T, Chai W-Y, Lin Y-J, Lin C-J, Chen P-Y, Tsai H-C, et al. Neuronavigation-guided focused ultrasound for transcranial blood-brain barrier opening and immunostimulation in brain tumors. Sci Adv. 2021 Feb 5;7(6):eabd0772.

65. Sonabend AM, Stupp R. Overcoming the Blood-Brain Barrier with an Implantable Ultrasound Device. Clin Cancer Res. 2019 Jul 1;25(13):3750-2.

66. Wei K-C, Tsai H-C, Lu Y-J, Yang H-W, Hua M-Y, Wu M-F, et al. Neuronavigation-Guided Focused Ultrasound-Induced BloodBrain Barrier Opening: A Preliminary Study in Swine. AJNR Am J Neuroradiol. 2013 Jan;34(1):115-20.

67. Pouliopoulos AN, Kwon N, Jensen G, Meaney A, Niimi Y, Burgess MT, et al. Safety evaluation of a clinical focused ultrasound system for neuronavigation guided blood-brain barrier opening in non-human primates. Sci Rep. 2021 Jul 22;11(1):15043.

68. Pouliopoulos AN, Wu S-Y, Burgess MT, Karakatsani ME, Kamimura HAS, Konofagou EE. A clinical system for non-invasive blood-brain barrier opening using a neuronavigation-guided single-element focused ultrasound transducer. Ultrasound Med Biol. 2020 Jan;46(1):73-89.

69. Liu H-L, Tsai C-H, Jan C-K, Chang H-Y, Huang S-M, Li $\mathrm{M}-\mathrm{L}$, et al. Design and Implementation of a Transmit/Receive Ultrasound Phased Array for Brain Applications. IEEE Trans Ultrasonics, Ferroelectrics, and Frequency Control. 2018 Oct;65(10):1756-67.

70. Wang S, Samiotaki G, Olumolade O, Feshitan JA, Konofagou EE. Microbubble Type and Distribution Dependence of Focused Ultrasound Induced Blood Brain Barrier Opening. Ultrasound Med Biol. 2014 Jan;40(1):130-7.

71. Wu S-K, Chu P-C, Chai W-Y, Kang S-T, Tsai C-H, Fan C-H, et al. Characterization of Different Microbubbles in Assisting Focused Ultrasound-Induced Blood-Brain Barrier Opening. Sci Rep. 2017 Apr 20;7(1):46689.

72. Ohshima M, Kamei S, Fushimi H, Mima S, Yamada T, Yamamoto T. Prediction of Drug Permeability Using In Vitro BloodBrain Barrier Models with Human Induced Pluripotent Stem Cell-Derived Brain Microvascular Endothelial Cells. BioResearch open access. 2019;8(1):2-209.

73. Roux GL, Jarray R, Guyot A-C, Pavoni S, Costa N, Théodoro F, et al. Proof-of-Concept Study of Drug Brain Permeability 
Between in Vivo Human Brain and an in Vitro iPSCs-Human Blood-Brain Barrier Model. Sci Rep. 2019 Nov 5;9:16310.

74. Oksanen M, Petersen AJ, Naumenko N, Puttonen K, Lehtonen S̆, Gubert Olivé M, et al. PSEN1 Mutant iPSC-Derived Model Reveals Severe Astrocyte Pathology in Alzheimer's Disease. Stem Cell Reports. 2017 Dec 12;9(6):1885-97.

75. Hernández D, Rooney LA, Daniszewski M, Gulluyan L, Liang $\mathrm{HH}$, Cook AL, et al. Culture Variabilities of Human iPSCDerived Cerebral Organoids Are a Major Issue for the Modelling of Phenotypes Observed in Alzheimer's Disease. Stem Cell Rev Rep. 2021 Mar 16;

76. Katt ME, Mayo LN, Ellis SE, Mahairaki V, Rothstein JD, Cheng $\mathrm{L}$, et al. The role of mutations associated with familial neurodegenerative disorders on blood-brain barrier function in an iPSC model. Fluids and Barriers of the CNS. 2019 Jul 15;16(1):20.

77. Momcilovic O, Sivapatham R, Oron TR, Meyer M, Mooney $\mathrm{S}$, Rao MS, et al. Derivation, Characterization, and Neural Differentiation of Integration-Free Induced Pluripotent Stem Cell Lines from Parkinson's Disease Patients Carrying SNCA, LRRK2, PARK2, and GBA Mutations. PLoS One. 2016 May 18;11(5):e0154890.

78. Park T-E, Mustafaoglu N, Herland A, Hasselkus R, Mannix R, FitzGerald EA, et al. Hypoxia-enhanced Blood-Brain Barrier Chip recapitulates human barrier function and shuttling of drugs and antibodies. Nat Commun. 2019 Jun 13;10(1):2621.

79. Neal EH, Marinelli NA, Shi Y, McClatchey PM, Balotin KM, Gullett DR, et al. A Simplified, Fully Defined Differentiation Scheme for Producing Blood-Brain Barrier Endothelial Cells from Human iPSCs. Stem Cell Reports. 2019 Jun 11;12(6):1380-8.

80. Hollmann E, Bailey A, Potharazu A, Neely M, Bowman A, Lippmann E. Accelerated differentiation of human induced pluripotent stem cells to blood-brain barrier endothelial cells. Fluids and Barriers of the CNS. 2017;14(1):9.

81. Lu TM, Houghton S, Magdeldin T, Durán JGB, Minotti AP, Snead A, et al. Pluripotent stem cell-derived epithelium misidentified as brain microvascular endothelium requires ETS factors to acquire vascular fate. PNAS. $2021 \mathrm{Feb} 23$

82. Lippmann ES, Al-Ahmad A, Azarin SM, Palecek SP, Shusta EV. A retinoic acid-enhanced, multicellular human blood-brain barrier model derived from stem cell sources. Scientific Reports. 2014 Feb 24;4(1):4160.

83. Vatine GD, Barrile R, Workman MJ, Sances S, Barriga BK, Rahnama M, et al. Human iPSC-Derived Blood-Brain Barrier Chips Enable Disease Modeling and Personalized Medicine Applications. Cell Stem Cell. 2019 Jun 6;24(6):995-1005.e6.

84. Oikari LE, Pandit R, Stewart R, Cuní-López C, Quek H, Sutharsan R, et al. Altered Brain Endothelial Cell Phenotype from a Familial Alzheimer Mutation and Its Potential Implications for Amyloid Clearance and Drug Delivery. Stem Cell Reports. 2020;14(5):924-39.

85. Blanchard JW, Bula M, Davila-Velderrain J, Akay LA, Zhu L, Frank A, et al. Reconstruction of the human blood-brain barrier in vitro reveals a pathogenic mechanism of APOE4 in pericytes. Nat Med. 2020 Jun;26(6):952-63.

86. Du F, Yu Q, Chen A, Chen D, ShiDu YS. Astrocytes Attenuate Mitochondrial Dysfunctions in Human Dopaminergic Neurons Derived from iPSC. Stem Cell Reports. 2018 Jan 27;10(2):366-74.

87. di Domenico A, Carola G, Calatayud C, Pons-Espinal M, Muñoz JP, Richaud-Patin Y, et al. Patient-Specific iPSC-Derived Astrocytes Contribute to Non-Cell-Autonomous Neurodegeneration in Parkinson's Disease. Stem Cell Reports. 2019 Feb 12;12(2):213-29.
88. Pereira JD, DuBreuil DM, Devlin A-C, Held A, Sapir Y, Berezovski E, et al. Human sensorimotor organoids derived from healthy and amyotrophic lateral sclerosis stem cells form neuromuscular junctions. Nat Commun. 2021 Aug 6;12(1):4744.

89. Wong AD, Ye M, Levy AF, Rothstein JD, Bergles DE, Searson PC. The blood-brain barrier: an engineering perspective. Front Neuroeng. 2013 Aug 30;6:7.

90. Janowicz PW, Leinenga G, Götz J, Nisbet RM. Ultrasoundmediated blood-brain barrier opening enhances delivery of therapeutically relevant formats of a tau-specific antibody. Scientific reports. 2019;9(1):9255-9255.

91. Nisbet RM, Brici D, Janowicz P, Götz J. Characterization of novel tau-specific antibodies and enhancement of their delivery into the brain using focused scanning ultrasound. Alzheimer's \& Dementia: The Journal of the Alzheimer's Association. 2019;15(7):P1629-30.

92. Nisbet RM, Van Der Jeugd A, Leinenga G, Evans HT, Janowicz PW, Götz J. Combined effects of scanning ultrasound and a tauspecific single chain antibody in a tau transgenic mouse model. Brain. 2017;140(5):1220-30.

93. Liu Y, Gong Y, Xie W, Huang A, Yuan X, Zhou H, et al. Microbubbles in combination with focused ultrasound for the delivery of quercetin-modified sulfur nanoparticles through the blood brain barrier into the brain parenchyma and relief of endoplasmic reticulum stress to treat Alzheimer's disease. Nanoscale. 2020 Mar 19;12(11):6498-511.

94. Lin C-Y, Hsieh H-Y, Chen C-M, Wu S-R, Tsai C-H, Huang C-Y, et al. Non-invasive, neuron-specific gene therapy by focused ultrasound-induced blood-brain barrier opening in Parkinson's disease mouse model. Journal of Controlled Release. 2016 Aug 10;235:72-81.

95. Zhang H, Sierra C, Kwon N, Karakatsani M, Jackson-Lewis V, Przedborski S, et al. Focused-ultrasound Mediated Anti-AlphaSynuclein Antibody Delivery for the Treatment of Parkinson's Disease. 2018 IEEE International Ultrasonics Symposium (IUS). 2018;

96. Xhima K, Nabbouh F, Hynynen K, Aubert I, Tandon A. Non-invasive delivery of an $\alpha$-synuclein gene silencing vector with MR-guided focused ultrasound. Mov Disord. 2018 Oct;33(10):1567-79.

97. Wang F, Li N, Hou R, Wang L, Zhang L, Li C, et al. Treatment of Parkinson's disease using focused ultrasound with GDNF retrovirus-loaded microbubbles to open the blood-brain barrier. Open Chemistry. 2020 Jan 1;18(1):882-9.

98. Kovacs ZI, Kim S, Jikaria N, Qureshi F, Milo B, Lewis BK, et al. Disrupting the blood-brain barrier by focused ultrasound induces sterile inflammation. Proceedings of the National Academy of Sciences of the United States of America. 2017;114(1):E75-84.

99. Yang J, Kong C, Jia L, Li T, Quan M, Li Y, et al. Association of accelerated long-term forgetting and senescence-related bloodborne factors in asymptomatic individuals from families with autosomal dominant Alzheimer's disease. Alzheimer's Research \& Therapy. 2021 May 27;13(1):107.

100. Villeda SA, Luo J, Mosher KI, Zou B, Britschgi M, Bieri G, et al. The ageing systemic milieu negatively regulates neurogenesis and cognitive function. Nature. 2011 Sep;477(7362):90-4.

101. Pandit R, Leinenga G, Götz J. Repeated ultrasound treatment of tau transgenic mice clears neuronal tau by autophagy and improves behavioral functions. Theranostics. 2019;9(13):3754-67.

102. Fengler S, Kurkowsky B, Kaushalya SK, Roth W, Fava E, Denner P. Human iPSC-derived brain endothelial microvessels in a multi-well format enable permeability screens of anti-inflammatory drugs. 2021 May. Available from: https://www.biorxiv.org/ content/10.1101/2021.05.03.442133v1 
103. Salvador E, Shityakov S, Förster C. Glucocorticoids and endothelial cell barrier function. Cell Tissue Res. 2014;355(3):597-605.

104. Noumbissi ME, Galasso B, Stins MF. Brain vascular heterogeneity: implications for disease pathogenesis and design of in vitro blood-brain barrier models. Fluids and Barriers of the CNS. 2018;15(1):12-12.

105. Schaeffer S, Iadecola C. Revisiting the neurovascular unit. Nat Neurosci. 2021 Sep;24(9):1198-209.

106. Saunders A, Macosko E, Wysoker A, Goldman M, Krienen $\mathrm{F}$, de Rivera $\mathrm{H}$, et al. Molecular Diversity and Specializations among the Cells of the Adult Mouse Brain. Cell. 2018 Aug 9;174(4):1015-1030.e16.

107. Vanlandewijck M, He L, Mäe MA, Andrae J, Ando K, Del Gaudio F, et al. A molecular atlas of cell types and zonation in the brain vasculature. Nature. 2018;554(7693):475-80.

108. Lendahl U, Nilsson P, Betsholtz C. Emerging links between cerebrovascular and neurodegenerative diseases - a special role for pericytes. EMBO Rep. 2019 Nov 5;20(11):e48070.

109. Licht T, Sasson E, Bell B, Grunewald M, Kumar S, Kreisel T, et al. Hippocampal neural stem cells facilitate access from circulation via apical cytoplasmic processes. eLife. 2020;9.

110. El-Agamy SE, Abdel-Aziz AK, Esmat A, Azab SS. Chemotherapy and cognition: comprehensive review on doxorubicininduced chemobrain. Cancer Chemother Pharmacol. 2019 Jul;84(1):1-14.

111. Perez-Lloret S, Barrantes FJ. Deficits in cholinergic neurotransmission and their clinical correlates in Parkinson's disease. npj Parkinson's Disease. 2016 Feb 18;2(1):1-12.

112. Rodríguez JA, Reyes CJ, Gutiérrez MI, Vera A, Martínez-Fong $\mathrm{D}$, Negreira C, et al. Opening the Blood-Brain Barrier in the Substantia Nigra of Rat Brain with Focused Ultrasound and Microbubbles. In: 2019 Global Medical Engineering Physics Exchanges/ Pan American Health Care Exchanges (GMEPE/ PAHCE). 2019. p. 1-5.

\section{Authors and Affiliations}

\section{Joanna M. Wasielewska ${ }^{1,2}$ (D) Anthony R. White ${ }^{1,3}$ D}

1 Cell \& Molecular Biology Department, Mental Health Program, QIMR Berghofer Medical Research Institute, Brisbane, QLD, Australia

2 Faculty of Medicine, The University of Queensland, Brisbane, QLD, Australia
113. Pediaditakis I, Kodella KR, Manatakis DV, Le CY, Hinojosa CD, Tien-Street W, et al. Modeling alpha-synuclein pathology in a human brain-chip to assess blood-brain barrier disruption. Nat Commun. 2021 Oct 8;12(1):5907.

114. Devi G, Scheltens P. Heterogeneity of Alzheimer's disease: consequence for drug trials? Alzheimers Res Ther. 2018 Dec 19;10:122.

115. Young AL, Marinescu RV, Oxtoby NP, Bocchetta M, Yong K, Firth NC, et al. Uncovering the heterogeneity and temporal complexity of neurodegenerative diseases with Subtype and Stage Inference. Nat Commun. 2018 Oct 15;9(1):4273.

116. Vlahos K, Sourris K, Mayberry R, McDonald P, Bruveris FF, Schiesser JV, et al. Generation of iPSC lines from peripheral blood mononuclear cells from 5 healthy adults. Stem Cell Res. 2019 Jan;34:101380.

117. Okumura T, Horie Y, Lai C-Y, Lin H-T, Shoda H, Natsumoto B, et al. Robust and highly efficient hiPSC generation from patient non-mobilized peripheral blood-derived CD34+ cells using the auto-erasable Sendai virus vector. Stem Cell Research \& Therapy. 2019 Jun 24;10(1):185.

118. Deng J, Huang Q, Wang F, Liu Y, Wang Z, Wang Z, et al. The Role of Caveolin-1 in Blood-Brain Barrier Disruption Induced by Focused Ultrasound Combined with Microbubbles. Journal of Molecular Neuroscience. 2012;46(3):677-87.

119. Sheikov N, McDannold N, Jolesz F, Zhang Y-Z, Tam K, Hynynen K. Brain arterioles show more active vesicular transport of blood-borne tracer molecules than capillaries and venules after focused ultrasound-evoked opening of the blood-brain barrier. Ultrasound Med Biol. 2006 Sep;32(9):1399-409.

Publisher's Note Springer Nature remains neutral with regard to jurisdictional claims in published maps and institutional affiliations.

3 School of Biomedical Sciences Faculty of Medicine, The University of Queensland, Brisbane, QLD, Australia 